\title{
Towards Active Muscle Pattern Analysis for Dynamic Hand Motions via sEMG
}

Jiahan $\mathrm{Li}^{1}$, Yinfeng Fang ${ }^{2}$, Yongan Huang ${ }^{5}$, Gongfa $\mathrm{Li}^{1,3,4}$, Zhaojie $\mathrm{Ju}^{2}$ and Honghai $\mathrm{Liu}^{2}$

${ }^{1}$ Key Laboratory of Metallurgical Equipment and Control Technology, Wuhan University of Science and Technology, Ministry of Education, Wuhan 430080, China

${ }^{2}$ School of Computing, University of Portsmouth, Portsmouth PO1 3HE, UK

${ }^{3}$ Research Center for Biomimetic Robot and Intelligent Measurement and Control, Wuhan University of Science and Technology, Wuhan 430081, China

${ }^{4}$ Institute of Precision Manufacturing, Wuhan University of Science and Technology, Wuhan 430081, China

${ }^{5}$ State Key Lab of Digital Manufacturing Equipment and Technology Huazhong University of Science and Technology Wuhan, China

jiahan.li@foxmail.com

yinfeng.fang@port.ac.uk

yahuang@hust.edu.cn

ligongfa@wust.edu.cn

zhaojie.julport.ac.uk

honghai.liu@port.ac.uk

\begin{abstract}
Surface Electromyographys (sEMG) as a popular human-computer interaction method can reflect the activity of human muscles. When the human forearm finishes different hand motions, there will be strong sEMG signals in different regions of the skin surface. This paper investigates the mapping relationship between sEMG signal patterns and the dynamic hand motions. Four different hand motions are studied based on the extracted signal with mean absolute value (MAV) features and the shape-preserving piecewise cubic interpolation method. In the experiments, a 16-channel electrode sleeve is used to collect 9subject EMG signals. According to the distribution of electrodes in the forearm, the forearm surface is divided into 8 different muscle regions. The preliminary experimental results show that different hand motions can cause different distributions of sEMG signals in different regions. It confirms that different subjects show similar patterns for same motions. The experimental results can be applied as new sEMG features with a higher computational speed.
\end{abstract}

Keywords: sEMG, MAV, shape-preserving piecewise cubic interpolation, local maximum, muscle regions.

\section{$1 \quad$ Introduction}

sEMG has been widely used as the effective method of controlling prosthetic. sEMGbased hand movement classification and recognition is the main research direction. In 
order to get high classification rates, there are several methods and algorithms have been developed such as multichannel surface EMG system [1], adaptive directed acyclic graph [2], ANNs [3], neuro-fuzzy classifiers [4] and other methods.

sEMG is a kind of biological signal with complex noises. that are caused by inherent equipment and environment noise, electromagnetic radiation, motion artifacts, and the interaction of different tissues [5]. Many algorithms and features are proposed to optimize hand motion classification and recognition based on EMG signals. We adopted multiple features commonly used in previous studies such as mean absolute value, zero crossing, slope sign change, and waveform length, in the algorithm for extracting handposture features, and the k-nearest-neighbors (KNN) algorithm as the classifier to perform hand-posture recognition [6]. Feature extraction and feature classification are two important tasks after sEMG data acquisition [7]. In recent decades, numerous studies have investigated the features of EMG signals as well as machine learning methods [8]. Many advanced algorithms have also been introduced into the field of pattern recognition based on EMG signals such as GMM to handle the dimension-reduction problem for EMG missing-data compensation [9].

Many studies have focused on processing EMG signals only. Many researchers hope to apply sEMG to prosthetic hand control. In recent years, some researcher proposed a synchronous robot control system based on the sEMG signals of human upper limb motions [10]. Although sEMG technology improvements have been made over the years, the sEMG control is usually limited to opening and closing. The main reason for this is the lack of robustness of the prosthetic hand control method. The position of the sensors is one of the main factors influencing the sEMG signals and, as a consequence, control robustness [11]. In order to better get good motions classification results. Some researchers fuse EMG signals with other signals. Xiangxin Li et al research a motionclassification strategy based on sEMG-EEG signal combination for upper-limb amputees [12]. Ying Sun et al proposed a gesture recognition method based on kinect and sEMG signal fusion [13].

Recently, some researchers began to apply sEMG signals to biological and medical research. S. Moore and M.P. McGuigan research the functional wavelet resolution of the sEMG frequency spectrum to represent high and low frequency motor unit recruitment in human lower limb muscles. This suggests the same force was produced using different patterns of neuromuscular recruitment [14]. Jose E. Cavazos and Jonathan Halford use of EMG signal to inform GTC seizure semiology. They prove the duration of tonic and clonic phases of GTCS may be quantified from sEMG data recorded by the Brain Sentinel device [15]. Some researchers want to create patient-specific models to improve and objectify these predictions. The relationship between 3D lip movement and sEMG activities was accommodated in a state-space model [16]. Surface electromyography (sEMG) data acquired during lower limb movements have the potential for investigating knee pathology. Some researchers propose an ICA-EBM-Based sEMG classifier for recognizing lower limb movements in individuals with and without knee Pathology [17]. Some researchers develop a diagnosis evaluation system of facial nerve paralysis using sEMG [18]. These studies extend the field of EMG applications.

EMG is a technique used for evaluating and recording the activation signal of muscles and also utilized for the electrical manifestation of the contractions of muscles [19]. 
This paper based on sEMG studies the relationship between hand movements and muscle activity. At the same time, this paper proves the mapping relationship between the active muscle areas and the hand motions through the experimental results. Based on the experimental results, new EMG features can be developed later. The rest of this paper is organized as follows. Section II discusses the experimental data collection methods and experimental data analysis methods. Section III proves the mapping relationship between the active muscle areas and the hand motions through the experimental results. Section VI draws the conclusion, gives the discussion of future works.

\section{Data Collection and Processing}

\subsection{SEMG Signals Collection}

Nine subjects took part in the experiment. All of them were healthy able-bodied subjects, without any prior neuromuscular disorders (eight males and one female, aged 2030 , denoted as P1 to P9).

Experiments use a 16-channel electrode sleeve to collect experimental data. The electrodes are modified from standard disposable $\mathrm{Ag} / \mathrm{AgCl} \mathrm{ECG}$ electrodes by means of gel removing. Electrodes are evenly fixed on an elastic fabric in advance. The procedure of arranging the electrodes on the forearm is as simple as wearing a sleeve. An empty sleeve (without electrodes on it) covers the previous one to guarantee a proper electrode to skin impedance. Before wearing the electrodes sleeve, subjects are obliged to wash their forearm for the better electrode to skin impedance as well [1].

Electrode sleeve is worn in the subject's right hand to collect data. Subjects use the ulna as a reference to wearing the 16-channel electrode sleeve. The ulnar coincides with the midline of the connection of electrode 8 and electrode 16 and the connection of electrode 1 and electrode 9. Sleeve wear position is shown in Fig. 1.

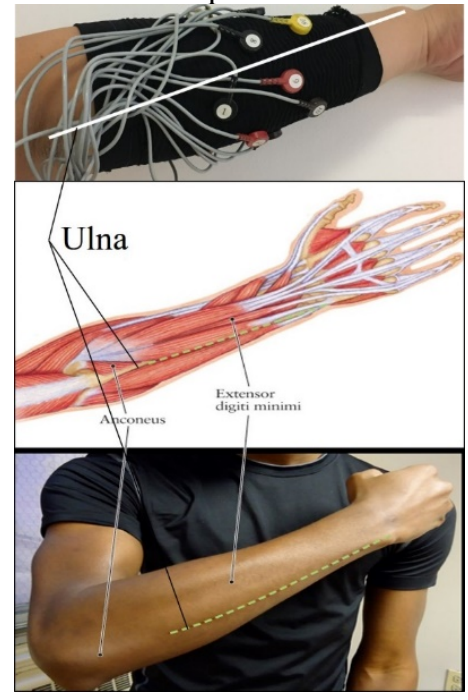

Fig. 1. The reference position to wearing the 16-channel electrode sleeve. 
Each subject wears the electrode sleeve for about 2 minutes. This operation can make the electrode fully contact with the skin to reduce signal noise. The experiment collects the sEMG signals of 4 different hand motions of each subject. The four motions were fist firstly and then turn left wrist, turn right wrist, turn the wrist up and down the wrist. Experimental motions are shown in Fig. 2. The subjects repeated each action 12 times. The experimenter needs to complete the hand motions within 3 seconds.

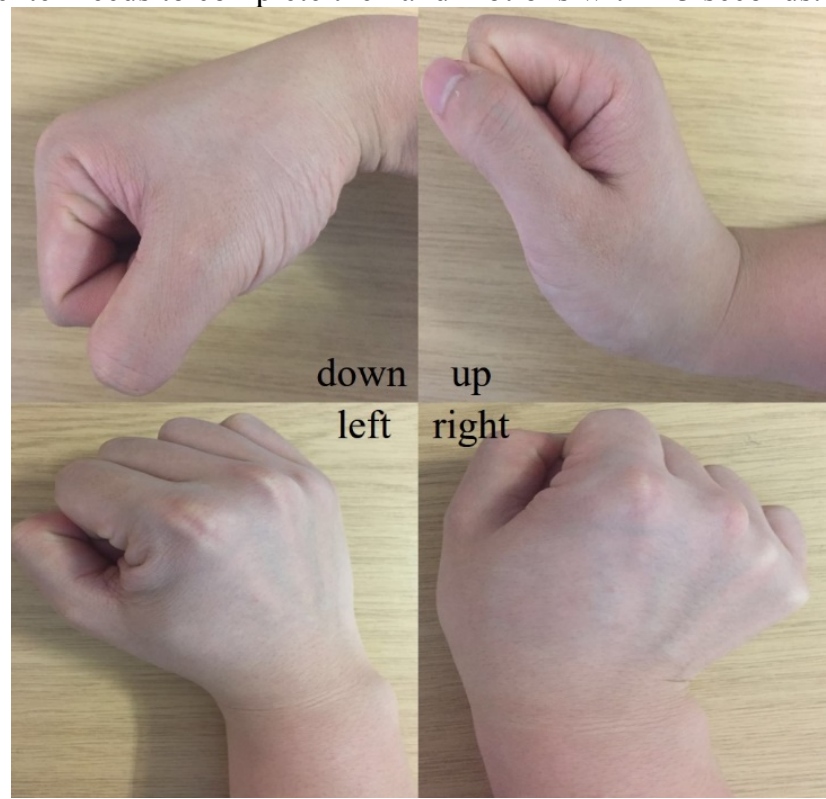

Fig. 2. The experimental motions.

\subsection{Processing of sEMG Signals}

In this paper, sEMG signal sampling frequency is set to $1 \mathrm{kHz}$, which is twice higher than the maximum frequency of sEMG signals. The newly received data with 16 channels are put into a buffer under a customized format. Once the buffer is full, a data parcel would be copied to a special buffer in the USB unit and prepared to be read by the USB host in a PC.

The original sEMG signals are filtered skin surface voltage. The subject's experimental motions are completed in 3 seconds. Every 3 seconds intercept a sEMG signal segment. Mean Absolute Value (MAV) is calculated for each signal segment. MAV is the average rectified value (ARV) and can be calculated using the moving average of full-wave rectified EMG. More specifically, it is calculated by taking the average of the absolute value of EMG signal. Since it represents the simple way to detect muscle contraction levels, it becomes a popular feature for myoelectric controlled applications. It is defined as (1). where $\mathrm{N}$ denotes the length of the signal and $\mathrm{xn}$ represents the EMG signal in a segment [5]. 


$$
M A V=\frac{1}{N} \sum_{N=1}^{N}\left|\mathrm{x}_{n}\right|
$$

Each channel in the subject's experimental motion will correspond to a MAV value. In this paper, we use the MAV value as the ordinate and the electrode number as the abscissa. This will get a lot of data points in the coordinate system. A subject data point of a motion shown in Fig. 3.

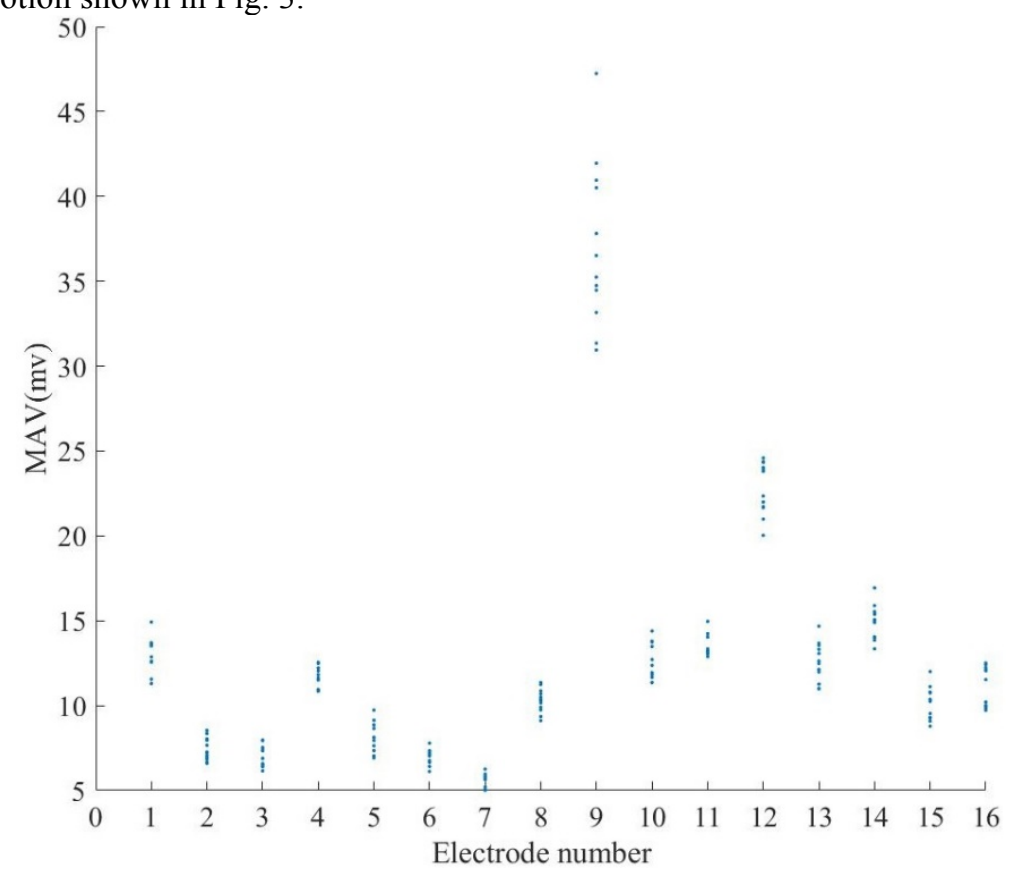

Fig. 3. A subject data point of a motion.

This paper uses shape-preserving piecewise cubic interpolation to fit data points. After fitting the data, a fitting curve is obtained. The shape-preserving piecewise cubic interpolation use a piecewise cubic polynomial $P(x)$. On each subinterval (2), the polynomial $P(x)$ is a cubic Hermite interpolating polynomial for the given data points with specified derivatives (slopes) at the interpolation points. $P(x)$ interpolates $y$, that is (3), and the first derivative $d p / d x$ is continuous. The second derivative $d^{2} p / d x^{2}$ is probably not continuous so jumps at the $x_{j}$ are possible. The cubic interpolant $P(x)$ is shape preserving. The slopes at the $x_{j}$ are chosen in such a way that $P(x)$ preserves the shape of the data and respects monotonicity. Therefore, on intervals where the data is monotonic. So is $P(x)$, and at points where the data has a local extremum, so does $P(x)$ [20]. One of the subjects of a motion to the curve shown in Fig. 4.

$$
X_{k} \leq X \leq X_{k+1}
$$




$$
P\left(x_{j}\right)=y_{j}
$$

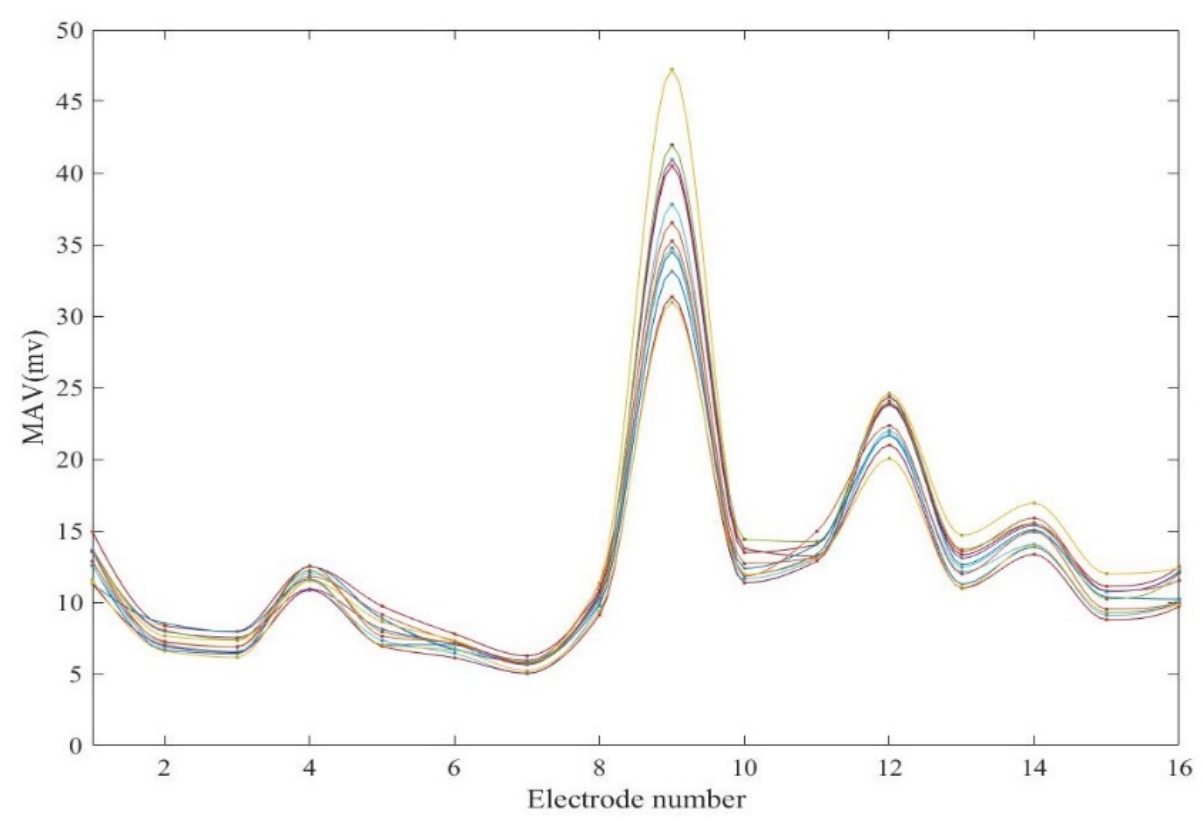

Fig. 4. One of the subjects of a motion to the curve.

\section{$3 \quad$ Pattern Analysis and Results}

A total of 36 datasets were collected in the experiment. The MAV is calculated for each dataset, and the corresponding curve is fitted using shape-preserving piecewise cubic interpolation. This paper calculates the local maximum on the curve. Each fitting curve represents the intensity of a SEMG between electrodes.

According to the distribution of the electrode sleeve and the human forearm surface muscle distribution, the mapping between the electrode and the muscle can be obtained. This paper will be on the surface of the forearm muscle to the two-dimensional plane. This method can intuitively show the positional relationship between the muscle and the electrode. The positional relationship between the muscle and the electrode shown in Fig. 5. The positional relationship is not completely accurate, and the experimental positional relationship will have a small deviation from the positional relationship in the Fig. 5. MAV represents the simple way to detect muscle contraction levels. According to the local maximum on the curve and positional relationship between the muscle and the electrode, it concludes that each subject has an active muscle area for each type of hand motion. 


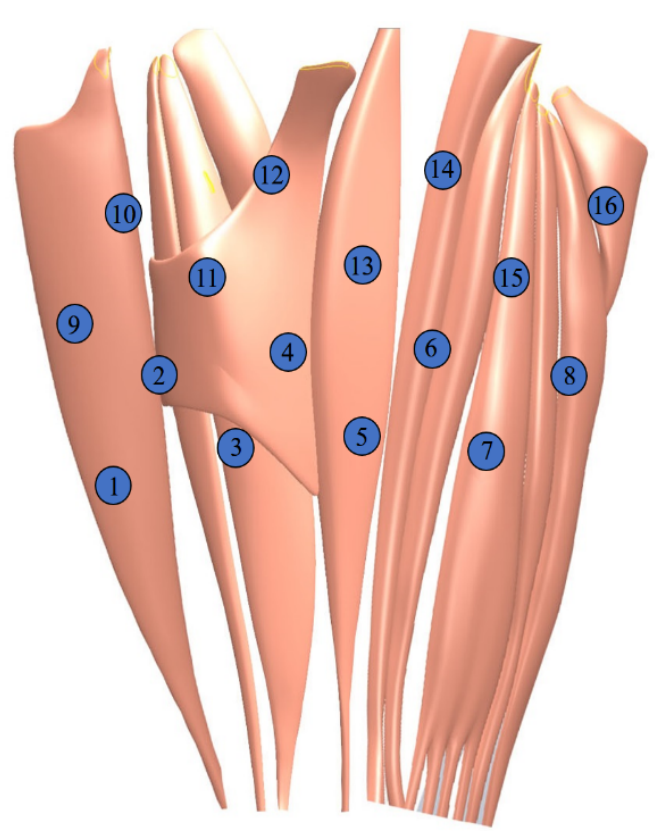

Fig. 5. Positional relationship between the muscle and the electrode.

In this paper, 36 datasets are calculated in turn. Active electrodes for each activity of each experimenter are counted. According to the statistical results, a total of 149 local maximum points was collected in the 4 experimental hand motions. Each local maximum point corresponds to one active electrode number. The statistical results (active electrodes number) are shown in Table 1.

Table 1. active electrodes number.

\begin{tabular}{ccccc}
\hline Motion & Up & Down & Left & Right \\
\cline { 1 - 4 } Subject & & & & \\
\hline P1 & $4,12,14$ & $4,6,8,13$ & $2,5,10,13$ & $4,9,12,14$ \\
P2 & $2,4,10,12,14$ & $4,6,8,10,14$ & $2,5,10,13$ & $4,6,9,12,14$ \\
P3 & $4,6,10,12,14$ & $4,6,12,14$ & $2,5,10,12,14$ & $3,10,12,14$ \\
P4 & $4,8,10$ & $5,8,11,14$ & $5,9,11,13$ & $4,8,11,14$ \\
P5 & $4,7,10,12,15$ & $4,6,7,10,12,15$ & $7,10,12,14$ & $4,7,9,12,14$ \\
P6 & $5,8,10,12,15$ & $4,8,10,12,15$ & $4,6,10,15$ & $5,9,13$ \\
P7 & $4,10,11,14$ & $4,7,10,14$ & $5,10,14$ & $9,10,14$ \\
P8 & $5,7,10,14$ & $2,10,4$ & $5,9,11,13,14$ & $5,9,11,14$ \\
P9 & $5,10,14$ & $3,5,10,14$ & $5,9,11,14$ & $5,9,11,14$ \\
\hline
\end{tabular}


Of the 149 local maximum points, there are 27 local maxima belonging to the up motion, 39 local maxima for the down motion, 37 local maxima for the left motion, and 36 local maxima for the right motion. The active electrode reflects the major role of the corresponding muscle in the motion. Combining the statistical results in Table I with the positional relationship between the muscle and the electrode map can give an active muscle area during the motion.

In this paper, Because the electrodes are distributed in pairs, the human upper arm muscle is divided into 8 regions. The area covered by a pair of longitudinal electrodes divides the muscle. Each muscle area covers 1 to 2 muscles. This paper will be the 8 areas number R1-R8. As shown in Fig. 6. The active electrodes of each action are matched to the positional relationship map.

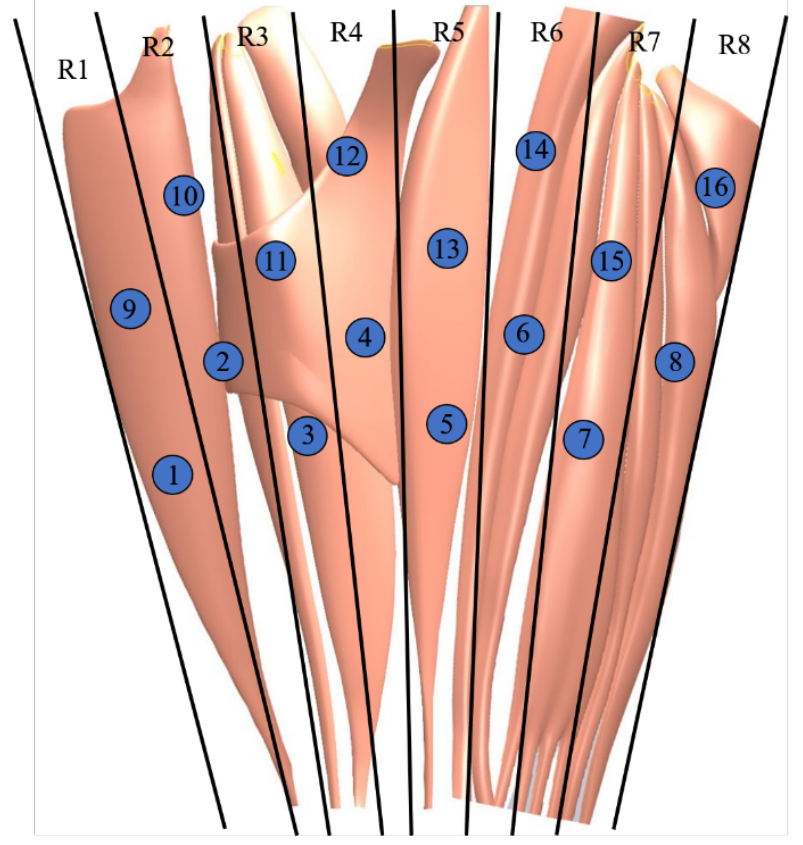

Fig. 6. R1-R8 muscle areas.

Due to the hand motions are done by muscle synergies, this paper selected 3 active muscle regions for each experiment motion. Based on preliminary observations of active electrode data. R2, R3, and R4 is the active area of up-hand motion. R4, R5, and $\mathrm{R} 6$ is the active area of down-hand motion. R2, R5 and R6 is the active area of lefthand motion. R1, R4, and R6 is the active area of right-hand motion. 21 up motion active electrodes were located in R2, R3 and R4 region. The amount of data in the R2, $\mathrm{R} 3$, and $\mathrm{R} 4$ region accounted for $57 \%$ of the total data for up motion. 22 down motion active electrodes were located in R4, R5, and R6 region. The amount of data in the R4, $\mathrm{R} 5$, and R6 region accounted for $56 \%$ of the total data for down motion. 26 left motion active electrodes were located in R2, R5, and R6 region. The amount of data in the R2, $\mathrm{R} 5$, and R6 region accounted for $70 \%$ of the total data for left motion. 24 left motion 
active electrodes were located in R1, R4, and R6 region. The amount of data in the R1, R4 and R6 region accounted for $67 \%$ of the total data for right motion. Active electrodes that are not in the active muscle area are also mostly located around the area. The matching rate of active electrode and muscle region are shown in Fig. 7.

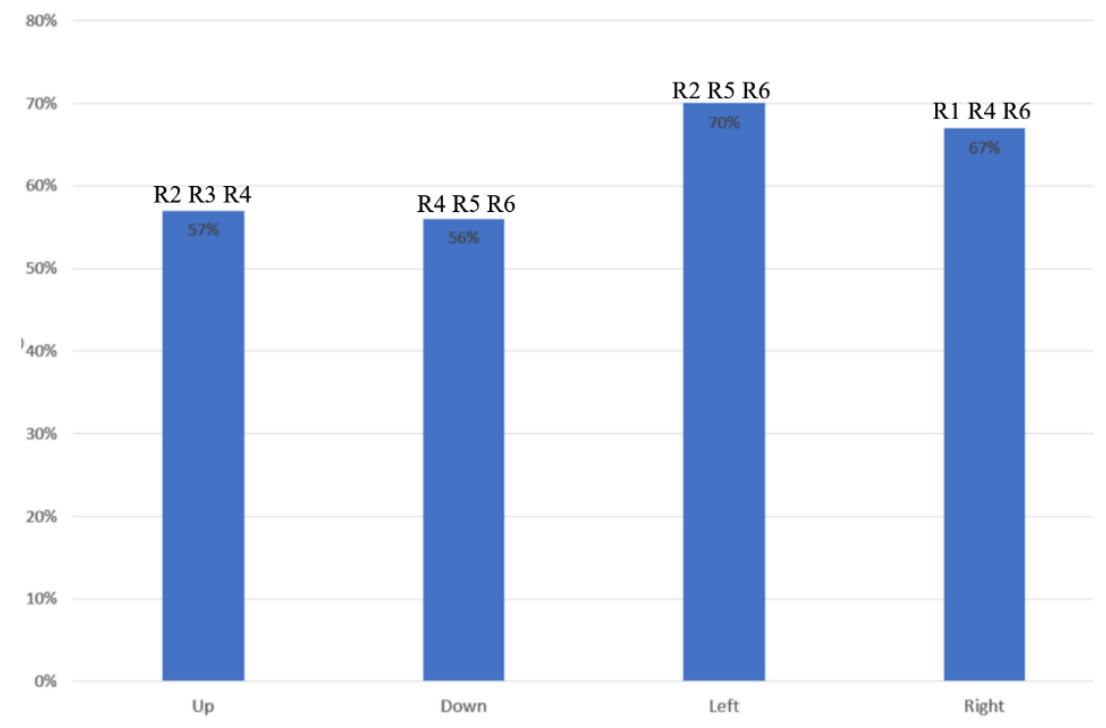

Fig. 7. The matching rate of active electrode and muscle region.

In this paper, the above results are combined with the support vector machine (SVM) classification algorithm. The active muscle region is used as the motion feature to classify the corresponding hand motion. The experimental results showed that the classification accuracy of the 4 hand motions reached $87 \%, 92 \%, 87 \%$ and $83 \%$ respectively (see Fig. 8).

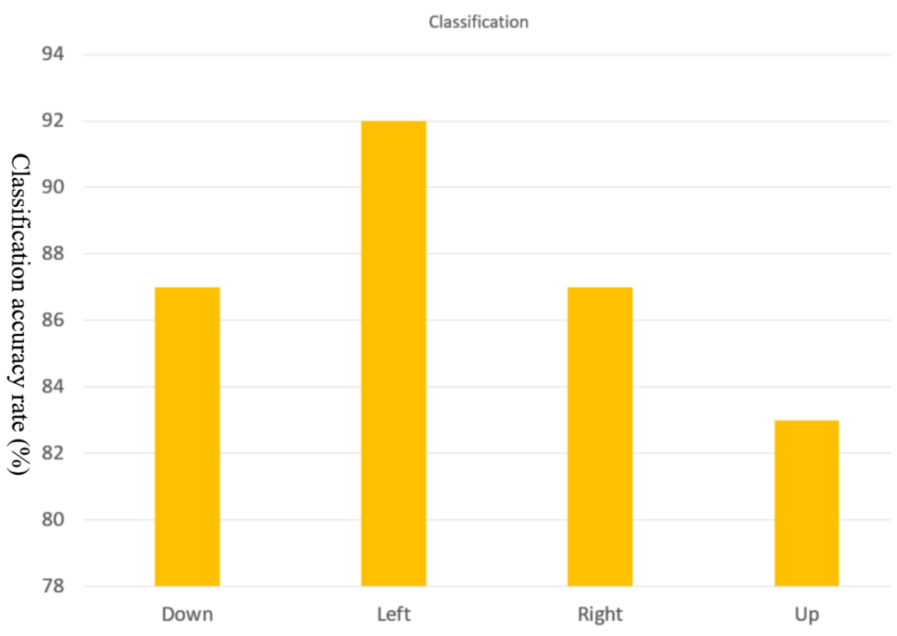

Fig. 8. The classification accuracy of the 4 hand motions 


\section{Conclusion and Future Work}

\subsection{Conclusion}

The research work has proved that the arm has different areas of muscle activity when performing different motions. There are some clear mappings between the active electrode areas and motions. When the subject finishes up motion, R2, R3, R4 muscle areas are more active. When the subject finishes the down motion, R4, R5, R6 muscle areas are more active. When the subject finishes left motions, R2, R5, R6 muscle areas are more active. When the subject finishes the right motion, R1, R4, R6 muscle area are more active. Subject motions and muscle active areas showed good mapping relations. These mappings can be used to improve the classification of the forearm motions as a new sEMG feature. The accuracy rate of hand motions recognition also proves it. On the other hand, this mapping can help medical researchers to research the muscular synergy problems through the sEMG signals.

\subsection{Future work}

There are many factors in the experiment that are unfavorable to the experimental results such as positional relationship between the muscle and the electrode map is not completely accurate; each subject's forearm thickness will affect positional relationship between the muscle and the electrode; subject sleeve wear position is not exactly accurate; the signal acquisition process will be influenced by some uncertainties. Experiments in the later stage can be improved to obtain more accurate experimental results. And more motions added to the experiment.

Improve the experimental results as a sEMG feature, using this feature to complete the classification of different hand motions. The computational method in this paper is concise, so it is expected that the classification speed of this feature will be superior to the traditional sEMG feature. Based on this experimental result, a real-time visualization system for muscle activity can be constructed. This visualization system is of great importance for the study of muscle activity and EMG-based hand motion recognition.

\section{Acknowledgment}

The authors would like to acknowledge the support from the Natural Science Foundation of China under Grant No. 51575412, 51575338 and 51575407, Natural Science Foundation of China Grant No.51575407, 51575338, 51575412 and 61733011, the EU Seventh Framework Programme (FP7)-ICT under Grant No. 611391, the Grants of National Defense Pre-Research Foundation of Wuhan University of Science and Technology (GF201705) and the Research Project of State Key Lab of Digital Manufacturing Equipment \& Technology of China under Grant No. DMETKF2017003. And this paper is funded by Wuhan University of Science and Technology graduate students' shortterm study abroad special funds. 


\section{References}

1. Yinfeng, F., Honghai, L., Gongfa, L., Xiangyang, Z.: A Multichannel Surface EMG System for Hand Motion Recognition. International Journal of Humanoid Robotics 12(2), 1550011 (2015).

2. Yaxu, X., Zhaojie, J., Jing, C., Honghai, L.: Multiple Sensors Based Hand Motion Recognition Using Adaptive Directed Acyclic Graph. Applied sciences 7(4), 358 (2017).

3. Rui, S., Rong, S., Kai-yu, T.: Complexity Analysis of EMG Signals for Patients after Stroke During Robot-Aided Rehabilitation Training Using Fuzzy Approximate Entropy. IEEE Transactions on Neural Systems And Rehabilitation Engineering 22(5), 1013-1019 (2013).

4. Khezri, M., Jahed, M., Sadati, N.: Neuro-fuzzy surface EMG pattern recognition for multifunctional hand prosthesis control. 2007 IEEE International Symposium on Industrial Electronics, pp. 269-274. Spain (2007).

5. Rezwanul, M., Ahsan, M., Ibrahimy, I., Othman, K.: Electromygraphy (EMG) Signal based Hand Gesture Recognition using Artificial Neural Network (ANN). 4th International Conference on Mechatronics. vol. 4, pp. 1-6. Malaysia (2015).

6. Wan-Ting, S., Zong-Jhe, L., Shih-Tsang, T., Tsorng-Lin, C., Chia-Yen, Y.: A bionic hand controlled by hand gesture recognition based on surface EMG signals:A preliminary study. Biocybernetics and biomedical engineering 38(1), 126-135 (2018).

7. Manea, S., Kamblib, R., Kazic, F., Singhc, N.: Hand Motion Recognition From Single Channel Surface EMG Using Wavelet \& Artificial Neural Network. Procedia Computer Science 49(1), 58-65 (2015).

8. An-Chih, T., Jer-Junn, L., Ta-Te, L.: A novel STFT-ranking feature of multi-channel EMG for motion pattern recognition. Expert Systems with Applications 42(7), 3327-3341 (2014).

9. Qichuan, D., Jianda, H., Xingang, Z., Yang, C.: Missing-Data Classification With the Extended Full-Dimensional Gaussian Mixture Model: Applications to EMG-Based Motion Recognition. IEEE Transactions on Industrial Electronics 62(8), 4994-5005 (2015).

10. Boyang, Z., Erwei, Y., Jun, J., Zongtan, Z.: A Synchronous Robot Control System Based on the sEMG Signals of Human Upper Limb Motions. Proceedings of the 36th Chinese Control Conference. pp. 5136-5140. China (2017).

11. Francesca, P., Matteo, C., Arjan, G., Henning, M.: Repeatability of grasp recognition for robotic hand prosthesis control based on sEMG data. 2017 International Conference on Rehabilitation Robotics (ICORR). pp. 17-20. UK (2017).

12. Xiangxin, L., Oluwarotimi, W., Samuel, X., Hui, Z.: A motion-classification strategy based on sEMG-EEG signal combination for upper-limb amputees. Journal of NeuroEngineering and Rehabilitation 14(1), 2-5 (2017).

13. Ying, S., Cuiqiao, L., Gongfa, L., Guozhang, J., Du, J., Honghai, L., Zhigao, Z.: Gesture Recognition Based on Kinect and sEMG Signal Fusion. Mobile Networks and Applications , (2018).

14. Moore, S., McGuigan, M.: Functional wavelet resolution of the sEMG frequency spectrum to represent high and low frequency motor unit recruitment in human lower limb muscles. International Journal of Science and Medicine in Sport 20, $73-75$ (2017).

15. Jose, E., Cavazos, J., Halford, M.: Use of sEMG to inform GTC seizure semiology. Neurology 88(2), 2-11 (2017).

16. Merijn, E., Maarten, A., Ludi, S., Dieta, B., Alfons, B., Ferdinand, H.: Predicting 3D lip movement using facial sEMG: a first step towards estimating functional and aesthetic outcome of oral cancer surgery. Med Biol Eng Comput, 55(4), 1-11 (2016).

17. Ganesh, N., Easter, S., Sridhar, P., Arjunan, A., Acharyya, D., Kumar, A.: Predicting 3D lip movement using facial sEMG: An ICA-EBM-Based sEMG Classifier for Recognizing 
Lower Limb Movements in Individuals With and Without Knee Pathology. IEEE Transactions on Neural Systems and Rehabilitation Engineering, 26(3), 675 (2018).

18. Shogo, O., Misaki, S., Hiroki, T., Takahiro, N.: Development of Diagnosis Evaluation System of Facial Nerve Paralysis Using sEMG. The 2017 International Conference on Artificial Life and Robotics. pp. 11893-11908. Japan (2017).

19. Yinfeng, F., Nalinda, H., Dalin, Z., Honghai, L.: Multi-modal Sensing Techniques for Interfacing Hand Prostheses: a Review. IEEE Sensors Journal, 15(11), 6065-6076 (2015).

20. Fritsch, F., Carlson, R.: Monotone piecewise cubic interpalation, SIAM Journal on Numerical Analysis. SIAM Journal on Numerical Analysis, 17(2), 238-246 (1980). 